\title{
The Rheumatoid Hand -An Orthopaedic Trainee's Current Treatment Perspective
}

\author{
Pratheek M Chikkalur ${ }^{1 *}$, Haydar Al Hussainy ${ }^{2}$, Richard Dias ${ }^{3}$, Tim McBride ${ }^{3}$ and Sukhrajbir Dhillon ${ }^{3}$ \\ ${ }^{1}$ Senior Clinical Fellow Trauma and Orthopaedics, The Royal Wolverhampton Hospitals NHS Foundation Trust, UK \\ ${ }^{2}$ Upper Limb Trauma \& Orthopaedic Fellow, The Royal Wolverhampton Hospitals NHS Foundation Trust, UK \\ ${ }^{3}$ Consultant Hand and Upper Limb Surgeon, New Cross Hospital, UK
}

Submission: February 16, 2018; Published: March 08, 2018

* Corresponding author: Pratheek M Chikkalur, The Department of Trauma \& Orthopaedic Surgery, The Royal Wolverhampton Hospitals NHS Foundation Trust, Wolverhampton road, Heath Town, Wolverhampton WV10 0QP, The United Kingdom, Tel: +447404495023; Email: pratheek.chikkalur@nhs.net

Abstract

The treatment of rheumatoid arthritis of the hand requires a holistic approach where surgical treatment is aimed at decreasing pain and improving function. This article briefly describes the principles and outlines the common procedures involved in treating rheumatoid hands.

Keywords: Rheumatoid arthritis; Rheumatoid Hand; Surgical Treatment; Current overview; Trainee

\section{Introduction}

With the introduction of newer biological medications to treat rheumatoid arthritis, the prevalence and severity of deformity seen clinically has decreased dramatically. There is a difference of opinion between hand surgeons and rheumatologists regarding the indication, timing, and effectiveness of surgery for the rheumatoid hand, especially since the evidence supporting the effectiveness of surgery for rheumatoid arthritis (RhA) is rather low [1]. For trainee surgeons, the exposure to rheumatoid hand surgery has drastically reduced giving that only a handful of surgeons regularly perform this type of surgery. A RhA patient with hand deformity does not necessarily equate to surgical intervention. It is important to understand patients' needs and expectations for improvement and available surgical options that predictably ameliorate function. Despite these shortcomings in outcomes-based research, surgery still remains a mainstay for many patients to improve hand function.

\section{Discussion}

The principles of surgical treatment of rheumatoid hand were benchmarked by William A Souter who set up the criteria for successful planning and execution of surgical treatment. The guiding principle was that the mere existence of deformity is not necessarily an indication for surgery as even in the presence of remarkable deformity and architectural disorganization, efficient function can be preserved with regards to power, grip, and pinch. The surgical programme needs to be tailored to the individual patient with aims of surgery being relief of pain, restoration of function, prevention of further damage and cosmetic improvement in decreasing order. In the final analysis advisability of any operation must be assessed in terms of the potential function improvement relative to individual patient needs [2].

Souter also developed the procedural order sequence according to the potential benefit and negative effects where he preferred extensor tendon synovectomy and excision of the ulna with wrist stabilisation if required as a most redeeming and gratifying operation. This was followed by flexor tendon synovectomy and metacarpophalangeal (MCP) joint arthroplasty to produce excellent results. In order to succeed such operations require collaboration with allied professionals like orthotists, hand therapists, occupational therapists, etc. The less rewarding surgeries were those of proximal interphalangeal (PIP) joint replacement and correction of finger deformity as it required advanced surgical expertise. Souter also suggested that thumb procedures should be carried out after reconstructing other fingers, thus allowing the thumb to be placed in an optimal functional position. He also believed that it is better to carry out several small operations at relatively frequent intervals rather than carrying out a major onslaught at once. This should allow adequate hand therapy, recovery time, and allow for dynamic or static deformities to settle and plan further procedures precisely and effectively [2]. This article will review the most common forms of surgery currently utilized for the management of RhA within the hand. 


\section{Orthopedics and Rheumatology Open Access Journal}

\section{MCP Joint}

RhA results in volar subluxation of the proximal phalanges and ulnar drift of the fingers. Inability to extend the fingers limits the mass grip function of hand, while fine pinch is hampered because the index and middle fingers can no longer oppose the thumb in a tip-to-tip pinch [3]. Fusion of the finger MCP joint is rarely performed because the arc of motion of the fingers is initiated at the MCP joint [4]. In the early stages of RhA, synovectomy of the MCP joint coupled with a cross intrinsic transfer in which the ulnar lateral bands are transferred to either the proximal phalanges or the extensor is performed [5].

In patients with chronic MCP joint subluxation or with joint destruction, an arthroplasty procedure is indicated. MCP joint arthroplasty has the advantage of shortening the skeleton at the MCP joint, which will decrease the tension on the tendons and ligaments contributing to the ulnar deformity. Silicone implants have continued to be popular for the RhA hand because of the ease of placement and the relative accessibility for implant replacement. A critical sequence in the operation is to centralize the extensor tendon by a combination of imbricating the stretched radial sagittal bands and releasing the tight ulnar bands. Standard protocol after this arthroplasty is to initiate a six-week dynamic extension exercise protocol [6].

\section{Tendon rupture and Tenosynovitis}

Synovitis is the hallmark of patients with RhA. This frequently affects extensor tendons of the hand more than the flexor counterparts [7]. The surgical management of tenosynovitis is tenosynovectomy, which is safe and effective in restoring function in patients with RhA. Besides, it can be useful in preventing tendon rupture and median nerve compression neuropathy [8]. Tendon rupture is a common dilemma in patients with $\mathrm{RhA}$. The definitive treatment for the tendon rupture is addressing the offending causes like removing the ulnar head and excising hypertrophic synovial tissue that can cause attrition and weakness of the extensor mechanism respectively [9].

Strategies for extensor tendon reconstruction follow the principles for tendon transfer. When the little finger extensor tendon is ruptured, the distal end of the intact tendon can be transferred end-to-side to the intact ring finger. When the ring and little finger extensor tendons are ruptured, the ideal transfer is to use the extensor indicis proprius (EIP) tendon to power the ring and little fingers. When the middle, ring, and little fingers are ruptured, transfer the Flexor Digitorum Superficialis (FDS) tendon from either the middle or the ring finger to power the ring and little fingers, while the EIP tendon is used to power the middle finger. Although synergistic with wrist extension; using FDS for finger extension is not a synergistic transfer that might prove hard for some patients to accommodate to postoperatively. The FDS is recommended to pass either through the ulnar or radial subcutaneous tunnels to avoid potential scarring from tunnelling through the interosseous membrane [10]. Beware that some RhA patients have a zigzag hand and fingers deformity where the entire hand is radially deviated while the fingers are in an ulnar swept deformity.

\section{Finger Deformities: Boutonniere versus Swan-Neck}

The joints in the hand and wrist are linked, and the deformity of a proximal joint will affect the position of the joints distally. Swan-neck deformity usually causes a functional problem because the patient is unable to make a full fist due to the hyperextended posture of the PIP joint, whereas in Boutonnière's deformity, particularly in the ulnar digits, the concern is more of an aesthetic than a functional concern [6]. The aetiology of Boutonnière deformity arises at the PIP joint where synovitis causes an elongation of the central slip. The lateral bands sublux below the axis of rotation resulting in shortening of the retinacular ligaments. These constellations of events result in an incomplete extension of the PIP joint and hyperextension of the DIP joint due to tightening of the lateral bands. For flexible deformity, the treatment is soft tissue reconstruction along with joint synovectomy, tightening of the stretched central tendon and bringing the lateral band dorsally. For fixed deformity, the treatment is joint fusion or implant arthroplasty [6].

In swan-neck deformities, the treatment will be determined by where the problem originates because this deformity can arise from the Distal Interphalangeal (DIP) joint, the PIP joint, or the MCP joint. Synovitis of the MCP joint causes alteration of the volar plate causing MCP joint subluxation. At the end, it produces a shortening of the intrinsic muscles leading to PIP joint hyperextension. Moreover, synovitis of the MCP joint produces an alteration of the insertion of the long extensors on the dorsal base of the proximal phalanx, producing hyperextension of the PIP joint. The deformity of the PIP joint is the result of the transfer of the extensor force to the base of the middle phalanx. Synovitis of the PIP joint can extend to FDS tendons and joint collateral ligaments. This synovitis produces degeneration of the FDS insertion and of the volar plate and collateral ligaments. It ultimately determines abnormal hyperextension of the PIP joint because of the action of the extensor forces $[11,12]$. Synovitis of the DIP joint produces the rupture of the terminal extensor tendon insertion resulting in mallet finger deformity.

Deformities of the DIP joint are managed with DIP fusion. When a flexible hyperextension deformity of the PIP joint is present, the most accredited surgical option considered is tenodesis, which can be performed using the FDS [13] or the conjoint lateral band [14]. After the treatment of the PIP joint, it is crucial to treat both MCP and DIP joint deformities, which may contribute to hyperextension of the PIP joint [13]. In patients with severe damage of the PIP joint (as in articular cartilage damage, unstable dislocation, and irreducible hyperextension deformity), arthroplasty or fusion is indicated.

\section{Thumb Deformities}

It is said that the thumb constitutes $50 \%$ of the hand function. Therefore, the most rewarding part of rheumatoid hand surgery is to reconstruct the thumb so that the patient has a stable and 
supple thumb to improve grip strength, posture, and appearance. For Boutonniere thumb deformity, the MCP joint is the problem and fusion of the MCP joint will shorten the thumb a little, which will balance the extensor mechanism to give the thumb a more natural posture. For the swan-neck thumb, the problem is at the carpometacarpal (CMC) joint, for which one can either fuse the CMC joint or perform an arthroplasty procedure with either a tendon interposition or an implant [6].

\section{Conclusion}

Despite both rheumatologists and hand surgeons having a better armamentarium with newer drugs, design of implants, and surgical trickery; collaboration between them is paramount for the successful management of rheumatoid hand challenges. Rheumatologists serve as coordinators for the overall care of RhA patients treated by various specialists. A referral to hand surgeon does not mean failure of medical treatment but an introduction of the patient to a hand surgeon who can plan current and future care. In order to ascertain the long term effectiveness of hand surgery procedures on RhA patients there is a need for high impact clinical studies that could guide us all to the ideal management solutions.

\section{References}

1. Burke FD, Miranda SM, Owen VMF, Bradley MJ, Sinha S (2011) Rheumatoid hand surgery: differing perceptions amongst surgeons, rheumatologists, and therapists in the UK. J Hand Surg Eur Vol 36(8): 632-641.

2. Souter WA (1979) Planning treatment of the rheumatoid hand. Hand 11(1): 3-16.
3. Albright JA, Nagel DA (1967) Treatment of the rheumatoid hand: metacarpophalangeal joints. Connecticut Medicine 31(3): 178-182.

4. Roullet J, Chatin B (1973) Surgery of the metacarpophalangeal joints in chronic rheumatoid arthritis. Revue du Rhumatismeetdes Maladies Osteo-Articulaires 40(3): 214-215.

5. RM Curtis (1968) Treatment of the ulnar deviation of the metacarpophalangeal articulations in rheumatoid arthritis by pulling back the radial collateral ligament, Revue de Chirurgie Orthopediqueet Reparatrice de l'Appareil Moteur 54(4): 335-341.

6. Chung KC, Pushman AG (2011) Current Concepts in the Management of the Rheumatoid Hand. J Hand Surg Am 36(4): 736-747.

7. Gong HS, Lee JO, Baek GH, Kim BS, Kim JY (2012) Extensor tendon rupture in rheumatoid arthritis: a survey of patients between 2005 and2010 at five Korean hospitals. Hand surgery 17(1): 43-47.

8. LH Millender, EA Nalebuff (1975) Preventive surgery-tenosynovectomy and synovectomy. Orthop Clin North Am 6(3): 765-792.

9. Takhar G, Suthahar Y, Stratton R (2009) Rheumatoid hands, Clinical Medicine. Journal of the Royal College of Physicians of London 9(5): 498-499.

10. Chung US, Kim JH, Seo WS, Lee KH (2010) Tendon transfer or tendon graft for ruptured finger extensor tendons in rheumatoid hands. J Hand Surg Eur Vol 35(4): 279-282.

11. Harrison SH (1969) Rheumatoid deformities of the proximal interphalangeal joints of the hand. Ann Rheum Dis 28(5): 20-22.

12. Harrison SH (1971) The proximal interphalangeal joint in rheumatoid arthritis. Hand 3(2): 125-130.

13. Nalebuff EA (1989) The rheumatoid swan-neck deformity. Hand Clin 5(2): 203-214.

14. Thompson JS, Littler JW, Upton J (1978) The spiral oblique retinacular ligament (SORL). Journal of Hand Surgery 3(5): 482-487.

\section{Your next submission with Juniper Publishers will reach you the below assets}

- Quality Editorial service

- Swift Peer Review

- Reprints availability

- E-prints Service

- Manuscript Podcast for convenient understanding

- Global attainment for your research

- Manuscript accessibility in different formats

( Pdf, E-pub, Full Text, Audio)

- Unceasing customer service

Track the below URL for one-step submission https://juniperpublishers.com/online-submission.php 